\title{
APOBEC3B: Pathological Consequences of an Innate Immune DNA Mutator
}

\begin{abstract}
Michael B. Burns ${ }^{1,2,3}$, Brandon Leonard ${ }^{3,4,5,6}$, Reuben S. Harris S $^{3,4,5,7}$
Cancer is a disease that results from alterations in the cellular genome. Several recent studies have identified mutational signatures that implicate a variety of mutagenic processes in cancer, a major one of which is explained by the enzymatic activity of the DNA cytosine deaminase, APOBEC3B. As a deaminase, APOBEC3B converts cytosines to uracils in single-stranded DNA. Failure to properly repair these uracil lesions can result in a diverse array of mutations. For instance, DNA uracils can template the insertion of complementary adenines leading to $\mathrm{C}$-to-T transition mutations. DNA uracils can also be converted into abasic sites that, depending upon the DNA polymerase recruited to bypass this lesion in the template strand, can lead to adenine insertion and C-to-T mutations as well as cytosine insertion and C-to-G transversion mutations. Finally, DNA uracils can also be converted into DNA breaks

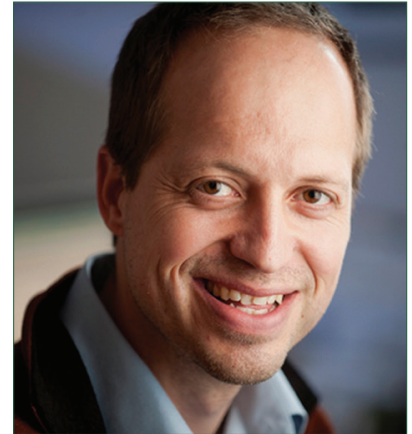

Prof. Reuben S. Harris that may precipitate some types of larger chromosomal aberrations observed in cancer. These studies cumulatively demonstrate that APOBEC3B is a major source of genetic heterogeneity in several human cancers and, as such, this enzyme may prove to be a critical diagnostic and therapeutic target. (Biomed J 2015;38:102-110)
\end{abstract}

\section{Key words: APOBEC3B, cancer, DNA cytosine deamination, genomic uracil, mutation}

$\mathrm{C}$ ancer is a genetic disease. For a malignancy to form and evolve, it needs to override the normal cellular safeguards encoded by the genome. Therefore, to prevent cancer initiation and limit its malignant potential, it is vital to understand the different sources of DNA damage and mutation that underlie this disease. ${ }^{[1]}$ Tumor genomes bear mutational signatures that reflect the underlying sources of those mutations. For instance, a mutation spectrum resulting from a combination of oxidative damage and a defect in DNA mismatch repair (MMR) will look different than a mutation signature resulting from oxidative damage alone. Research is underway to quantitatively deconvolute the multiple sources that give rise to these complex mutation patterns..$^{[2-4]}$ The sources of DNA damage and mutation in cancer can be classified into the general categories of exogenous and endogenous, where the exogenous sources are those that arise from the environment and the endogenous sources are those that arise from within the cell itself. Pyrimidine dimers that result from UV damage are a classic example of an exogenous agent generating lesions that lead predominantly to context-specific C-to- $\mathrm{T}$ transition mutations. ${ }^{[5,6]}$ Endogenous processes can be further categorized into passive and active sources of DNA damage and mutation. Passive mutation is characterized by a failure to repair DNA damage after it has occurred. Established sources of passive mutation are inherited deficiencies in DNA repair processes, such as MMR gene defects that are characterized molecularly by microsatellite DNA instability and clinically by predisposition to hereditary nonpolyposis colorectal cancer (HNPCC).

From the ${ }^{1}$ Genetics, Cell Biology, and Development Department, University of Minnesota, Minneapolis, MN, USA; ${ }^{2}$ Evolution, Ecology, and Behavior Department, University of Minnesota, Minneapolis, MN, USA; ${ }^{3}$ Masonic Cancer Center, University of Minnesota, Minneapolis, MN, USA; ${ }^{4}$ Institute for Molecular Virology, University of Minnesota, Minneapolis, MN, USA; ${ }^{5}$ Center for Genome Engineering, University of Minnesota, Minneapolis, MN, USA; ${ }^{6}$ Microbiology, Cancer Biology and Immunology Graduate Program, University of Minnesota, Minneapolis, MN, USA; ${ }^{7}$ Biochemistry, Molecular Biology and Biophysics Department, University of Minnesota, Minneapolis, MN, USA

Received: Jul. 09, 2014; Accepted: Nov. 26, 2014

Correspondence to: Prof. Reuben S. Harris, University of Minnesota, Biochemistry, Molecular Biology and Biophysics Department. 321 Church Street S.E., 6-155 Jackson Hall, Minneapolis, Minnestoa, 55455 USA. Tel: 1-612-6240457; Fax: 1-612-6252163; Email: rsh@umn.edu

DOI: $10.4103 / 2319-4170.148904$ 
Similarly, recombination repair gene defects can increase the rate of chromosomal aberrations and predispose to breast and ovarian cancers. ${ }^{[7]}$

Conversely, active endogenous sources of mutation are agents that directly damage DNA. Some of these sources are well known, such as oxidation of guanine and hydrolytic deamination of cytosine, but many others are poorly defined. ${ }^{[3,8]}$ However, much headway has been made recently in understanding additional endogenous sources of DNA damage, with our group ${ }^{[9-12]}$ and others ${ }^{[2,3,13,14]} \mathrm{im}$ plicating APOBEC3B, a member of the APOBEC family of single-stranded DNA (ssDNA) polynucleotide cytosine deaminases, in cancer genome mutagenesis [Figure 1].

\section{The APOBEC family}

The human APOBEC family includes 11 members: The namesake, Apolipoprotein B mRNA Editing enzyme, Catalytic subunit 1 (APOBEC1) on chromosome 6, APO$B E C 2$ on chromosome 12, APOBEC $3 A, B, C, D, F, G, H$ encoded in a tandem head-to-tail array on chromosome 22, APOBEC4 on chromosome 1, and Activation Induced Cytosine Deaminase (AID or AICDA) also on chromosome 6 [Figure 2A]. All of the APOBEC enzymes, except for APOBEC2 and APOBEC4, are capable of converting cytosine in ssDNA via a deamination reaction to uracil (C-to-U) [Figure 2B]. This water-mediated reaction can also occur spontaneously (as alluded above), though enzymatic deamination occurs at much faster rates especially on unprotected ssDNA substrates. ${ }^{[15]}$

With the exception of $A I D$, these genes were all named after $A P O B E C 1$ as a result of sequence homology indicating a shared lineage of inter- and intra-chromosomal gene duplication events. However, this nomenclature is misnomeric as $A P O B E C l$ is the only family member whose gene product carries out apolipoprotein B mRNA editing. ${ }^{[16-18]}$ In fact, many of these proteins have independent physiological functions. For example, AID is essential for both somatic hypermutation and class-switch recombination through deamination of variable and switch region DNA segments within rearranged immunoglobulin heavy and light chain genes. ${ }^{[19]}$

The APOBEC 3 proteins are also known to have at least three distinct physiologic functions in the human body, all of which are consistent with their role in innate immunity. First, many of the APOBEC 3s have been described to defend against a diverse array of viral pathogens, including retroviruses, hepatitis viruses, papillomaviruses, and others. ${ }^{[20-24]}$ Of note, APOBEC3D, F, G, and $\mathrm{H}$ have been shown to restrict HIV-1 replication by deaminating cDNA intermediates that normally occur during the HIV-1 life cycle. ${ }^{[25,26]} \mathrm{Sec}-$ ond, several APOBEC3s, including APOBEC $3 \mathrm{~A}, \mathrm{~B}$, and $\mathrm{F}$, have been shown to inhibit retrotransposition of L1 and Alu elements in human cells. ${ }^{[27-29]}$ Third, researchers have demonstrated that APOBEC $3 \mathrm{~A}$ and other family members have the potential to mediate the clearance of foreign DNA through a deamination-dependent mechanism. . $^{[7,30,31]}$

Recent work has also indicated that there are regions of the genome that appear more susceptible to mutation than others. Specifically, late-replicating and heterochromatic regions of the genome appear enriched for mutations when compared to early replicating and euchromatic DNA, respectively. ${ }^{[32,33]}$ In the case of late-replicating regions of the genome, one explanation for the increased mutation content is that these regions are more likely to harbor stalled replication forks, more ssDNA intermediates, and consequently more substrates for APOBEC3B. In the case of heterochromatic DNA, bearing in mind that ssDNA is the substrate for APOBEC3 deamination, heterochromatin is poorly transcribed and, thus, less accessible to DNA repair machinery than euchromatic regions. ${ }^{[34]}$ Moreover, highly transcribed regions of the genome are intrinsically less likely to tolerate mutations due to its essential nature (i.e. negative selection).

\section{Technical achievements}

The main challenge to assessing the role of APOBEC family members in cancer is that all of the family members share large amounts of sequence homology due to relatively recent gene duplication and divergence events. ${ }^{[35]}$ While a few APOBEC monoclonal antibodies $(\mathrm{mAb})$ have been generated, most lack the intended specificity in practice by binding multiple family members. This makes quantitative determination of the presence or absence of endogenous expression of individual family members, using currently available antibody reagents, very difficult at the protein level.

Even at the mRNA level, several of the APOBECs are highly similar. For example, $A P O B E C 3 A$ and the $\mathrm{C}$-terminal

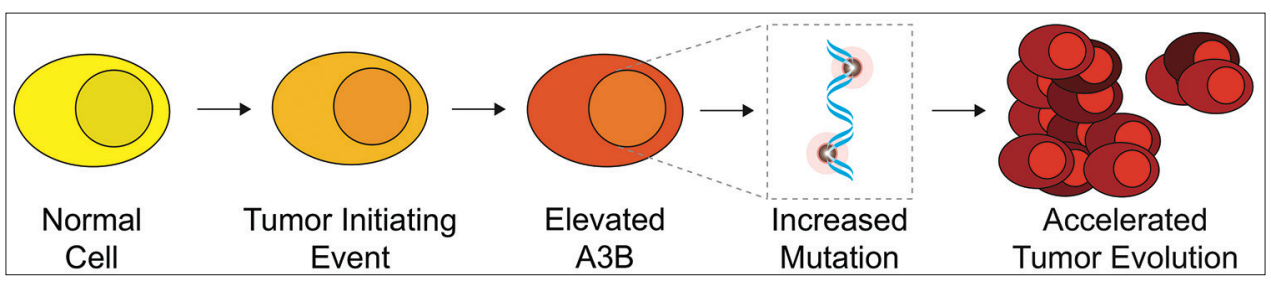

Figure 1: Model for APOBEC3B-driven tumor evolution. Upregulation of APOBEC3B in nascent cancer cells or during cancer development increases mutation rates and drives tumor evolution. 


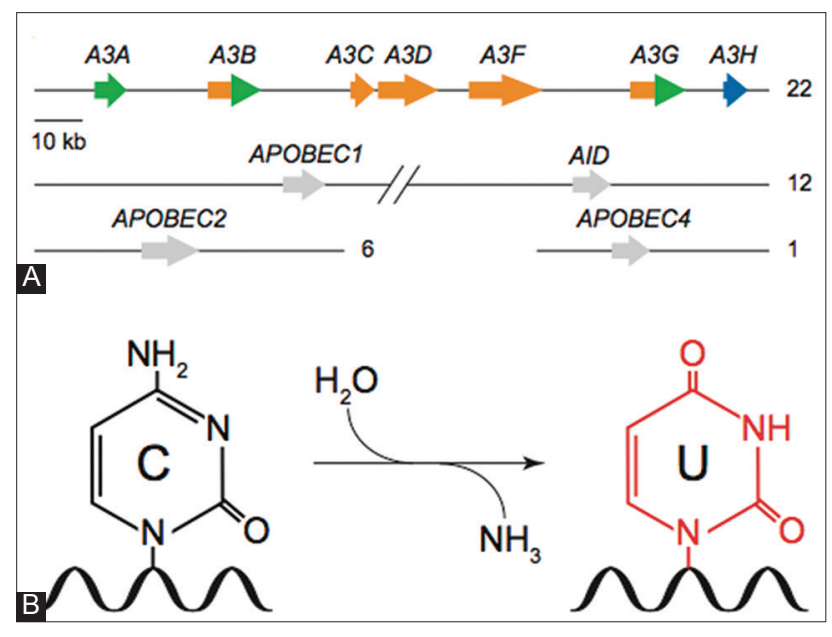

Figure 2: Introduction to the APOBEC family. (A) Depiction of the spatial organization of the APOBEC family members, with the $A P O B E C 3$ genes arrayed in tandem on chromosome 22, APOBEC1 and AID located on chromosome 12, and $A P O B E C 2$ and $A P O B E C 4$ encoded on chromosomes 6 and 1, respectively. (B) APOBEC3 family enzymes catalyze the hydrolytic reaction of cytosine to uracil in single-stranded DNA.

domain of $A P O B E C 3 B$ share $>90 \%$ nucleotide identity. Because of this identity, many of the current techniques to study global changes in mRNA expression are complicated by cross-hybridization issues. Many of the probes used to detect mRNA levels in microarrays are too short to discretely distinguish between the different $A P O B E C 3$ transcripts and often share homology between multiple family members. ${ }^{[9]}$ The longer reads generated by RNAseq and utilization of paired-end sequencing improves upon this issue, but even these are potentially susceptible to inappropriate read mapping. Fortunately, researchers have been able to construct and validate panels of reverse transcription quantitative PCR (RT-qPCR) assays that can be used to specifically quantify each individual APOBEC transcript. ${ }^{[36,37]}$ Overall, any method used to detect $A P O B E C 3$ expression must be designed and carefully validated to ensure specificity and efficiency.

\section{Previously implicated APOBECs}

The expression of APOBEC1 in transgenic animals was one of the first experiments that investigated whether APOBEC deamination can lead to cancer causing mutations. ${ }^{[38]}$ Transgenic expression of rabbit APOBEC1 in mice resulted in universal liver dysplasia, many of which progressed to hepatocellular carcinoma. While rabbit APOBEC1 clearly has a dramatic carcinogenic effect when expressed constitutively in transgenic mice, this was not the case when expressed in rabbits ${ }^{[38]}$ and it has not yet proven relevant to human cancers (although a recent study has implicated APOBEC1 in esophageal adenocarcinomas). ${ }^{[39]}$ It should also be noted that this original APOBEC1 study ${ }^{[38]}$ was performed prior to the discovery of AID/APOBEC catalyzed DNA cytosine deamination, ${ }^{[17,40]}$ and therefore, the authors inferred that off-target RNA editing caused the observed malignancies.

Several APOBEC3 family members have also been hypothesized to play a role in cancer since the initial discovery that they use DNA as a substrate. ${ }^{[17]}$ At that time, the difficulty in differentiating among the many family members made it unclear which, if any, family members might be driving mutation in cancer. Recently, it was reported that APOBEC 3G contributes to metastasis in hepatocellular carcinoma, though the research neither proposed nor tested a mechanistic explanation of the observation. ${ }^{[41]}$ More recently, using the aforementioned RT-qPCR assays, it has been concluded that APOBEC $3 \mathrm{G}$ is not currently a suspect in cancer onset or progression as abnormal levels of APOBEC3G have not been found in human cancer tissue when specific assays are applied. There is a chance that the normal level of APOBEC3G expressed in a given tissue may be misregulated at post-transcriptional levels, but again, there has been no evidence presented to support this hypothesis.

Because AID is known to deaminate genomic DNA as part of its normal physiological activity, it is easy to imagine that this protein may have detrimental off-target effects. Indeed, body-wide expression of murine AID in mice leads to premature death due to T-cell lymphomas and lung adenocarcinomas. ${ }^{[42]}$ In addition, AID is known to produce well-characterized carcinogenic chromosomal translocations as a side-effect of class-switch recombination. ${ }^{[43]}$ For example, AID is required for the chromosomal translocation between $c-m y c$ and the immunoglobulin $(I g)$ locus, which is associated with Burkitt's lymphoma. ${ }^{[4]]}$ These experiments provide proof of principle and a starting place from which to pursue research on the potential role of the other family members in cancer.

\section{$A P O B E C 3 B$ and breast cancer}

Since the discovery of the DNA mutating properties of the APOBEC family members in 2002, it has been hypothesized that one or more of them has a direct role in cancer mutagenesis. ${ }^{[17]}$ In the process of testing this hypothesis and identifying a cancer-related APOBEC, several criteria must be met. The implicated APOBEC must be expressed in the cancer, have access to genomic DNA, be catalytically active, and result in detectable changes to genomic DNA.

Burns et al. were the first to clearly identify APO$\mathrm{BEC} 3 \mathrm{~B}$ as the APOBEC family member at work in human cancer. ${ }^{[9]}$ They quantified the full repertoire of $A P O B E C$ family mRNA species in human breast cancer tissues and cell lines. These data showed that $A P O B E C 3 B$ was preferentially and specifically upregulated in a majority of the samples tested. This allowed subsequent efforts to be focused on elucidating the molecular mechanism by which 
this enzyme might operate in breast cancer. APOBEC3B is the only family member that constitutively localizes to the cell nucleus. ${ }^{[9,45,46]}$ Also, it retains deamination activity, increases the steady-state level of uracil in the cell's genome, and correlates with increased mutation, as determined by selection and enrichment techniques (thymidine kinase (TK)-fluctuation assay and 3D-PCR/sequencing). ${ }^{[9]}$ These findings indicated that in a large proportion of breast cancer cell lines, APOBEC3B is driving mutations that diversify the genetic landscape.

The key translation of these mechanistic studies to primary patient tumor genomes was the recognition that APOBEC3B deaminates ssDNA at a preferred sequence context. Biochemical assays in vitro demonstrated that APOBEC3B prefers substrate cytosines in 5"TCA and 5"TCG contexts ${ }^{[9,11]}$ Mutation data from three independent primary breast tumor genome datasets clearly indicated that mutations at these sites are significantly enriched. ${ }^{[9]}$ Moreover, $A P O B E C 3 B$ expression levels correlated positively with both cytosine mutation and overall mutation loads..$^{[9]}$

\section{Global analyses of mutation signatures}

The work by Burns and colleagues opened the door to larger scale genomic studies aimed at examining the contribution of APOBEC 3B to the mutation load across many different tumor types. ${ }^{[10,47]}$ These analyses revealed that $A P O B E C 3 B$ is significantly upregulated in many tumor types relative to its expression in normal tissue derived from the same organ. Furthermore, the cancer types expressing the highest levels of $A P O B E C 3 B$ also contained the most mutations. The most striking findings came when these groups examined the sequence context of the mutated cytosine bases (i.e. the trinucleotide motifs including the bases immediately 5' and 3' of each mutated cytosine). Here, several tumor types showed a mutation profile similar to that of recombinant APOBEC3B. Together, the data produced independently by the Harris and Gordenin labs suggest that APOBEC $3 \mathrm{~B}$ contributes most significantly to mutation in six distinct types of cancer: Bladder, cervix, lung (adenocarcinoma and squamous cell carcinoma), head and neck, and breast. Additional studies looking at general mutation patterns, though non-specific in implicating a particular APOBEC family member, have arrived at similar conclusions. ${ }^{[2,3,33,48-50]}$

\section{Mutagenic outcomes of genomic uracil}

One major challenge to determining which mutations directly result from APOBEC3B cytosine deamination events is understanding how genomic uracils are processed in cancer cells. It is established, based on prior research on AID, that U: G mispairs resulting from cytosine deamination can result in all six base substitution mutation types. ${ }^{[19]}$ While many $\mathrm{U}: \mathrm{G}$ lesions are likely repaired in an error-free manner by the canonical base excision repair pathway, lesions that escape this process have multiple distinct mutagenic potentials [Figure 3]. Briefly, the general steps required for repair include excision of the uracil lesion, nicking of the DNA backbone, and subsequent reincorporation of the correct nucleotides (light blue region in Figure 3).${ }^{[1]}$ At several of the steps required for repair, there are alternative outcomes that may lead to a variety of mutation types. Simple DNA replication across uracilated DNA results in C-to-T transitions (tan region in Figure 3), mutagenic MMR at $\mathrm{U}$ : $\mathrm{G}$ mispairs may result in transitions and/or transversions (green region in Figure 3), translesion DNA synthesis across abasic sites can result in transition mutations (yellow region in Figure 3), and finally, in highly deaminated regions, the repair process may generate nicks on both strands of the DNA double helix that are relatively close to one another potentially resulting in double-stranded breaks (purple region in Figure 3).

In breast cancer, Burns, Lackey, and colleagues found that $A P O B E C 3 B$ upregulation correlated with increased levels of transition mutations, suggesting that a proportion of the genomic uracils created by APOBCE3B either persist through DNA synthesis or are generated at a high enough rate that they are detectable in non-replicated DNA. As indicated above, if a uracil is not excised by a DNA glycosylase prior to DNA replication, it will template as a thymine and base pair with adenosine. After a subsequent round of DNA replication, the result is a C-to-T transition mutation. A similar result will occur if the genomic uracil is removed by uracil excision repair and an adenine is inserted opposite of the resulting abasic site during local DNA synthesis or replication. While these are perhaps the simplest mutational outcomes of cytosine deamination, many other pathways have been investigated. In fact, a recent paper by Leonard and colleagues has found a significant correlation between $A P O B E C 3 B$ upregulation and both C-to-A and C-to-G transversion mutations in whole genome sequences from 16 early-stage serous ovarian carcinomas. ${ }^{[11]}$ The authors suggested that the observed mutations may be the result of error-prone translesion DNA synthesis past non-instructional abasic sites created by uracil excision.

The above mechanisms are supported not only by the aforementioned work on AID and APOBEC3B, but also by more recent publications aimed at elucidating the proteins involved in the repair of APOBEC-mediated damage in yeast. One study has shown that a deficiency in uracil DNA glycosylase (UNG) or the translesion DNA polymerase, REV1, results in a mutation spectrum greatly skewed toward C-to-T transition mutations (at the expense of transversions), particularly in the presence of an active DNA cytosine deaminase. ${ }^{[14]}$ Another study used a panel of translesion 


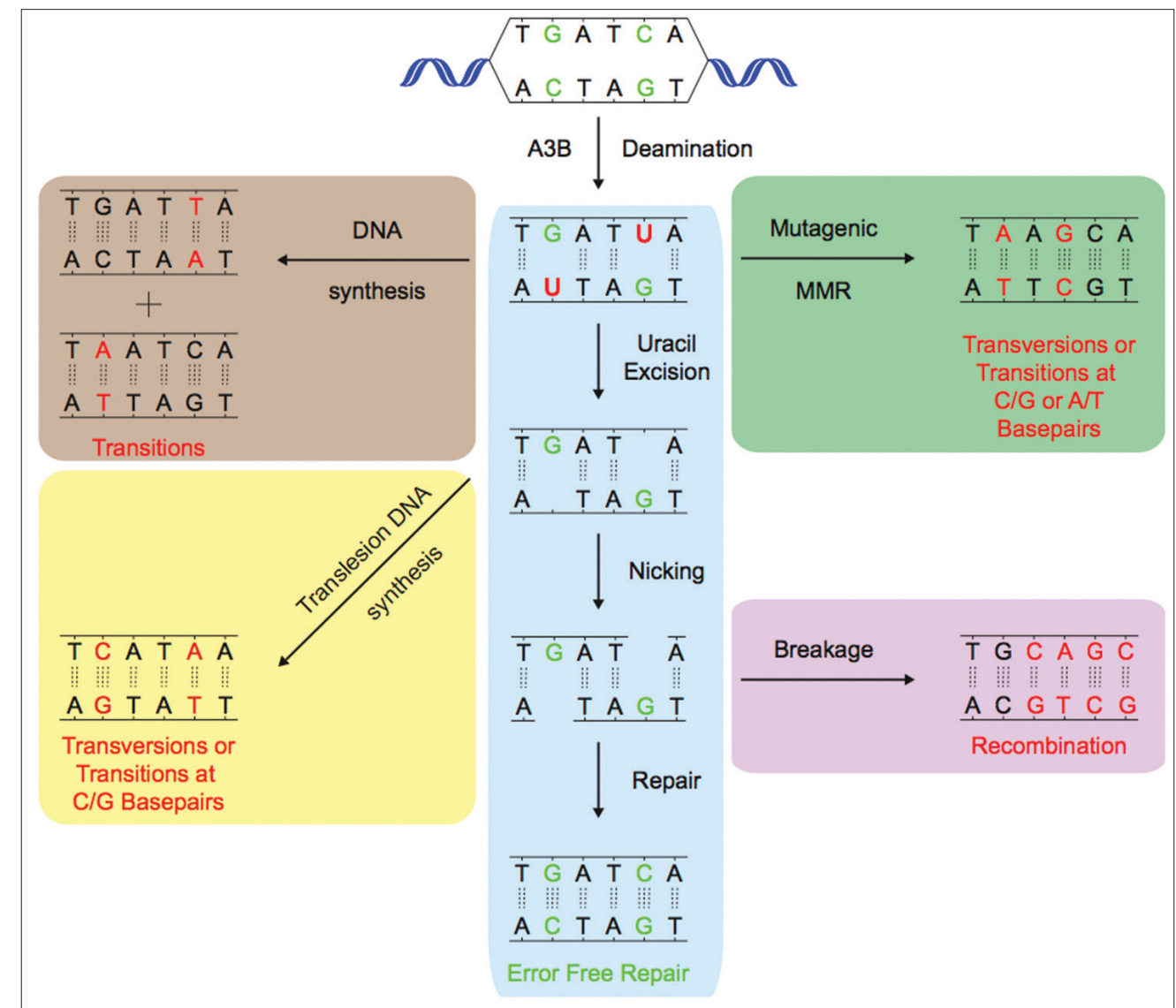

Figure 3: Potential mutagenic outcomes of uracil lesions. Canonical base excision repair is the most prevalent pathway and often results in error-free repair of uracil lesions (blue), but at least three intermediates in this process can lead to mutagenic outcomes. Uracils persisting through DNA synthesis can result in C-to-T transition mutations (tan). Mismatch repair can also process genomic uracils (as U/G mispairs) and result in mutations at the site of deamination as well as nearby bases during long patch repair (green). Translesion synthesis across abasic sites can lead to C-to-T transitions or to C-to-G or C-to-A transversions (yellow). Finally, the accumulation of nicks can lead to single- (not shown) and double-stranded breaks and genetic recombination. Double-stranded breaks can result when nicks occur on opposite strands of the helix within close proximity or when a replication fork hits a single nick (purple). Model adapted from Leonard and coworkers. ${ }^{[1]}$

polymerase mutants to show that REV1 and REV3 contribute most significantly to the formation of transversion mutations in yeast. ${ }^{[52]}$ This research provides support for the proposed models, but more studies are needed to identify the mechanisms at play in human tumors (with many more DNA polymerases than yeast) and to determine how other processes, such as MMR, recombination, and cell cycle checkpoints, might also influence mutagenic outcomes.

\section{An APOBEC3B deletion alelle}

Several studies have examined the APOBEC $3 B$ locus in human populations as part of both general surveys and more specific cancer studies. ${ }^{\left[{ }^{[3-56]}\right.}$ These analyses have identified an $A P O B E C 3 B$ deletion polymorphism circulating in the human population with an allelic frequency ranging from approximately 1 to $93 \%$, dependent upon the biogeographical ancestry of the population examined [Figure 4]. ${ }^{[53,57]}$ One group used a small Japanese cohort ( $<50$ patients) to assess breast cancer incidence and the $A P O B E C 3 B$ dele- tion polymorphism and found a statistically insignificant trend toward an inverse correlation between $A P O B E C 3 B$ and breast cancer. ${ }^{[54]}$ Two other groups used much larger cohorts to assess the relationship between the deletion allele and breast cancer incidence. ${ }^{[55,56]}$ These larger studies determined that there was a significant increase in the $A P O$ $B E C 3 B$ deletion allele among women with breast cancer. Unlike the Japanese study that collected data on the deletion allele frequency from normal healthy patients recruited into their study, these groups relied on data from the 1000 genomes project to determine the frequency of the deletion allele within their cohorts. ${ }^{[54-57]}$ These findings argue that $A P O B E C 3 B$ is somehow a protective factor, reducing the incidence of breast cancer in the populations studied. These observations are intriguing and may reflect compromised innate immune defenses, with increased levels of viral infection and endogenous retrotransposition expected in the absence of this enzyme. ${ }^{[29,54-57]}$

The $A P O B E C 3 B$ deletion allele may be protective with 


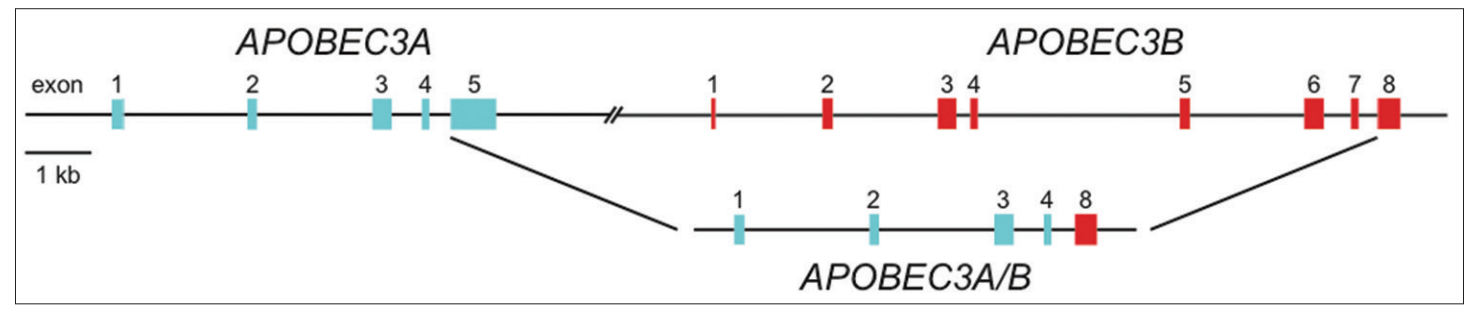

Figure 4: $A P O B E C 3 B$ deletion allele. A germline deletion between homologous regions of $A P O B E C 3 A$ and $A P O B E C 3 B$ has resulted in a chimeric gene in which exon 4 of $A P O B E C 3 A$ is fused to exon 8 of $A P O B E C 3 B$.

respect to breast cancer incidence; however, it was recently shown that once the cancer has formed, patients with estrogen receptor (ER)-positive tumors and $A P O B E C 3 B$ upregulation have significantly worse outcomes relative to those that lack expression of the enzyme. ${ }^{[12]}$ One of several cohorts in this study represented patients who had only been treated by surgical resection, indicating that $A P O B E C 3 B$ expression levels alone can provide a prognostic indication. This recent study is important because it clearly distinguishes incidence ("catching cancer") from progression (what happens once a cancer has been diagnosed). Additional studies on breast and other APOBEC3B-linked tumor types are needed to confirm and extend these initial findings.

There have been conflicting reports in the literature related to the potential contributions of different APOBEC3 family members to cancer mutagenesis, specifically, APOBEC3A and APOBEC3B. ${ }^{[2,3,9-11,14,46]}$ An argument has been presented that an APOBEC $3 \mathrm{~A} / \mathrm{B}$ mutation pattern persists even in cancer samples that harbor the $A P O B E C 3 B$ deletion allele. ${ }^{[58]}$ This, were it the case, would imply that the signature is likely the result of APOBEC3A since APOBEC3B is absent in the cells homozygous for the $A P O B E C 3 B$ deletion. Unfortunately, the only study to-date addressing this possibility failed to segregate the $A P O B E C 3 B$ deletion allele heterozygotes from the homozygotes and, thus, confounded interpretations by including as the majority of their "APOBEC $3 B$ deletion" samples, tumors with upregulated, active APOBEC $3 B{ }^{[58]}$ This, coupled with the finding that APOBEC3A when expressed endogenously is confined to myeloid lineage cell types, and the protein itself is located in the cytoplasm and is non-genotoxic, indicating that APOBEC3B remains the leading culprit for cancer mutagenesis. ${ }^{[31,36,37,46,59,60]}$ Further work is needed to determine unambiguously whether other APOBEC family members might contribute to cancer mutagenesis and, if so, then deduce their contributions relative to those of APOBEC3B.

\section{Connection to antiviral innate immunity}

A key question is how APOBEC3B becomes upregulated in cancer. Original studies eliminated many possibilities including gene amplification, chromosome translocation, promoter mutations, and other cis events such as differential methylation. ${ }^{[9]}$ Although there are likely to be many answers to this question, one possible clue comes from the strong link between $A P O B E C 3 B$ expression and mutagenesis in cervical and head/neck cancers ${ }^{[3,9,47]}$ and the fact that many of these cancers are human papillomavirus (HPV) driven. ${ }^{[61]}$ Henderson and colleagues recently demonstrated a clear segregation of exogenous, smoking-related mutations and APOBEC3B-driven mutations in head/neck cancers. ${ }^{[50]}$ Their findings further indicated that $A P O B E C 3 B$ upregulation correlated with HPV-positive status. Thus, for these cancer types, there may be a HPV-mediated mechanism of $A P O B E C 3 B$ upregulation. The mechanism (s) for APOBEC3B upregulation is less obvious for non-viral cancer types and is likely to require many future investigations.

\section{Conclusions}

There is a growing consensus in the field that APO$\mathrm{BEC} 3 \mathrm{~B}$ is a major and previously unappreciated source of mutation in several different cancer types. This enzyme is likely to contribute significantly to genetic, and thus phenotypic, heterogeneity within the tumors in which it is expressed. The full clinical significance of this conclusion has yet to be realized fully, but it may be relevant to diagnosis, prognosis, and ultimately therapy (e.g. ref. ${ }^{[12]}$ ).

Knowledge of APOBEC3B mutagenesis provides a framework for future therapeutic strategies. The most direct method to limit the impact of APOBEC3B impact would be to inhibit the enzyme's deaminase activity using small molecules and thereby to create a hypomutator state in the tumor [Figure 5]. The goal of this strategy would be to post-operatively (once tumor burden decreases) slow the rate of evolution of the remaining tumor cells, decrease the likelihood of resistance mutations arising, and ultimately render the remaining tumor cells more sensitive to conventional therapeutics. Alternatively, the as-yet-unknown pathways that drive APOBEC3B expression could be targeted to decrease the expression levels, highlighting the importance of further research on this topic. Conversely, as has been done for BRCA1/2-mutant cancers, DNA repair pathways could be modulated in an attempt to make APOBEC3B-dependent damage toxic, 


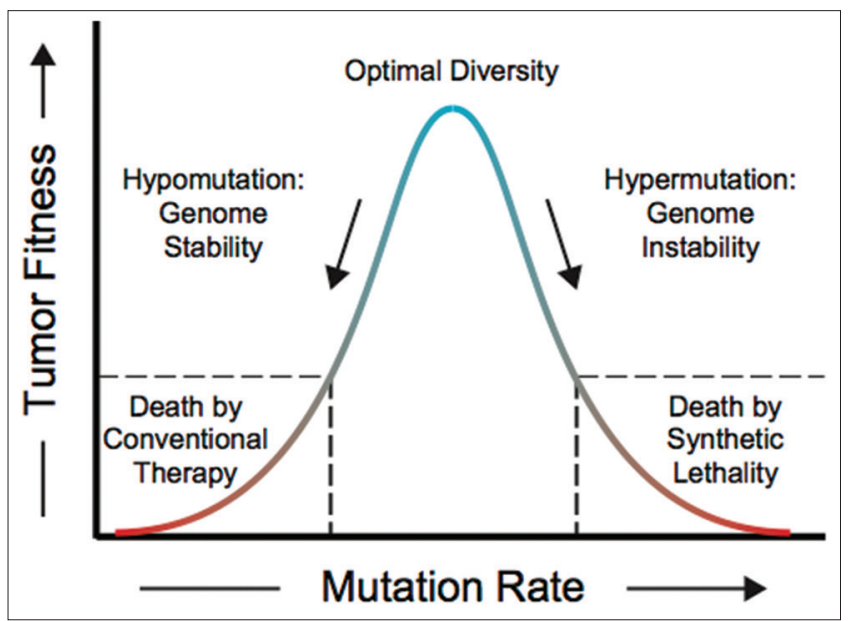

Figure 5: Therapeutic implications of APOBEC3B mutagenesis. Decreasing or increasing the mutation rate of APOBEC3B-expressing cells is predicted to result in decreased tumor fitness and tumor cell death. Decreasing the mutation rate through APOBEC3B inhibition may result in genome stabilization and a lower probability of drug resistance mutations (i.e. increased durability of conventional chemotherapeutics). Alternatively, increasing the level of APOBEC3B mutagenesis (directly by increasing APOBEC 3B levels or indirectly by creating a synthetic lethal state) may result in toxic levels of genomic lesions and tumor death. Adapted from similar concepts proposed for APOBEC3 mutagenesis of the retrovirus HIV-1. ${ }^{[62]}$

creating a hypermutator phenotype and a synthetic lethal state for tumor cells [Figure 5]. In either the hypomutation or hypermutation scenarios, the goal is to translate this basic research discovery into successful clinical results that improve and extend the lives of patients.

\section{Acknowledgments}

Cancer mutation work in the Harris lab has been supported by grants from the Department of Defense (CDMRP Idea Award BC121347), the Jimmy V Foundation for Cancer Research, the Minnesota Ovarian Cancer Alliance, the Norwegian Centennial Chair Program, and the Randy Shaver Cancer Research and Community Fund.

\section{REFERENCES}

1. Hanahan D, Weinberg RA. Hallmarks of cancer: The next generation. Cell 2011;144:646-74.

2. Nik-Zainal S, Alexandrov LB, Wedge DC, Van Loo P, Greenman CD, Raine K, et al. Mutational processes molding the genomes of 21 breast cancers. Cell 2012;149:979-93.

3. Alexandrov LB, Nik-Zainal S, Wedge DC, Aparicio SA, Behjati S, Biankin AV, et al. Signatures of mutational processes in human cancer. Nature 2013;500:415-21.

4. Alexandrov LB, Stratton MR. Mutational signatures: The patterns of somatic mutations hidden in cancer genomes. Curr Opin Genet Dev 2014;24:52-60.

5. Berger MF, Hodis E, Heffernan TP, Deribe YL, Lawrence MS,
Protopopov A, et al. Melanoma genome sequencing reveals frequent PREX2 mutations. Nature 2012;485:502-6.

6. Wei X, Walia V, Lin JC, Teer JK, Prickett TD, Gartner J, et al. Exome sequencing identifies GRIN2A as frequently mutated in melanoma. Nat Genet 2011;43:442-6.

7. King MC, Marks JH, Mandell JB; New York Breast Cancer Study Group. Breast and ovarian cancer risks due to inherited mutations in BRCA1 and BRCA2. Science 2003;302:643-6.

8. Barnes DE, Lindahl T. Repair and genetic consequences of endogenous DNA base damage in mammalian cells. Annu Rev Genet 2004;38:445-76

9. Burns MB, Lackey L, Carpenter MA, Rathore A, Land AM, Leonard $\mathrm{B}$, et al. APOBEC3B is an enzymatic source of mutation in breast cancer. Nature 2013;494:366-70.

10. Burns MB, Temiz NA, Harris RS. Evidence for APOBEC3B mutagenesis in multiple human cancers. Nat Genet 2013;45:977-83.

11. Leonard B, Hart SN, Burns MB, Carpenter MA, Temiz NA, Rathore A, et al. APOBEC3B upregulation and genomic mutation patterns in serous ovarian carcinoma. Cancer Res 2013;73:7222-31.

12. Sieuwerts AM, Willis S, Burns MB, Look MP, Gelder ME, Schlicker A, et al. Elevated APOBEC3B Correlates with Poor Outcomes for Estrogen-Receptor-Positive Breast Cancers. Horm Cancer 2014;5:405-13.

13. Shinohara M, Io K, Shindo K, Matsui M, Sakamoto T, Tada K, et al. APOBEC3B can impair genomic stability by inducing base substitutions in genomic DNA in human cells. Sci Rep 2012;2:806.

14. Taylor BJ, Nik-Zainal S, Wu YL, Stebbings LA, Raine K, Campbell PJ, et al. DNA deaminases induce break-associated mutation showers with implication of APOBEC $3 \mathrm{~B}$ and $3 \mathrm{~A}$ in breast cancer kataegis. Elife 2013;2:e00534.

15. Ehrlich M, Norris KF, Wang RY, Kuo KC, Gehrke CW. DNA cytosine methylation and heat-induced deamination. Biosci Rep 1986;6:387-93.

16. Conticello SG. The AID/APOBEC family of nucleic acid mutators. Genome Biol 2008;9:229.

17. Harris RS, Petersen-Mahrt SK, Neuberger MS. RNA editing enzyme APOBEC1 and some of its homologs can act as DNA mutators. Mol Cell 2002;10:1247-53.

18. LaRue RS, Andrésdóttir V, Blanchard Y, Conticello SG, Derse D, Emerman M, et al. Guidelines for naming nonprimate APOBEC3 genes and proteins. J Virol 2009;83:494-7.

19. Di Noia JM, Neuberger MS. Molecular mechanisms of antibody somatic hypermutation. Annu Rev Biochem 2007;76:1-22.

20. Vieira VC, Soares MA. The role of cytidine deaminases on innate immune responses against human viral infections. BioMed Res Int 2013;2013:683095.

21. Koito A, Ikeda T. Apolipoprotein B mRNA-editing, catalytic polypeptide cytidine deaminases and retroviral restriction. Wiley Interdiscip Rev RNA 2012;3:529-41.

22. Schmitz KM, Petersen-Mahrt SK. AIDing the immune system-DIAbolic in cancer. Semin Immunol 2012;24:241-5.

23. Harris RS, Hultquist JF, Evans DT. The restriction factors of human immunodeficiency virus. J Biol Chem 2012;287:40875-83.

24. Refsland EW, Harris RS. The APOBEC3 family of retroelement

Biomed J Vol. 38 No. 2

March - April 2015 
restriction factors. Curr Top Microbiol Immunol 2013;371:1-27.

25. Hultquist JF, Lengyel JA, Refsland EW, LaRue RS, Lackey L, Brown WL, et al. Human and rhesus APOBEC3D, APOBEC3F, $\mathrm{APOBEC} 3 \mathrm{G}$, and $\mathrm{APOBEC} 3 \mathrm{H}$ demonstrate a conserved capacity to restrict Vif-deficient HIV-1. J Virol 2011;85:11220-34.

26. Refsland EW, Hultquist JF, Harris RS. Endogenous origins of HIV-1 G-to-A hypermutation and restriction in the nonpermissive $\mathrm{T}$ cell line CEM2n. PLoS Pathog 2012;8:e1002800.

27. Carpenter MA, Li M, Rathore A, Lackey L, Law EK, Land AM, et al. Methylcytosine and normal cytosine deamination by the foreign DNA restriction enzyme APOBEC3A. J Biol Chem 2012;287:34801-8.

28. Bogerd HP, Wiegand HL, Doehle BP, Lueders KK, Cullen BR. $A P O B E C 3 A$ and $A P O B E C 3 B$ are potent inhibitors of LTR-retrotransposon function in human cells. Nucleic Acids Res 2006;34:89-95.

29. Stenglein MD, Harris RS. APOBEC3B and APOBEC3F inhibit L1 retrotransposition by a DNA deamination-independent mechanism. J Biol Chem 2006;281:16837-41.

30. Bulliard Y, Narvaiza I, Bertero A, Peddi S, Röhrig UF, Ortiz M, et al. Structure-function analyses point to a polynucleotide-accommodating groove essential for APOBEC3A restriction activities. J Virol 2011;85:1765-76

31. Stenglein MD, Burns MB, Li M, Lengyel J, Harris RS. APOBEC3 proteins mediate the clearance of foreign DNA from human cells. Nat Struct Mol Biol 2010;17:222-9.

32. Liu L, De S, Michor F. DNA replication timing and higher-order nuclear organization determine single-nucleotide substitution patterns in cancer genomes. Nat Commun 2013;4:1502.

33. Lawrence MS, Stojanov P, Polak P, Kryukov GV, Cibulskis K, Sivachenko A, et al. Mutational heterogeneity in cancer and the search for new cancer-associated genes. Nature 2013;499:214-8.

34. Adam S, Polo SE. Blurring the line between the DNA damage response and transcription: The importance of chromatin dynamics. Exp Cell Res 2014;329:148-53.

35. LaRue RS, Jónsson SR, Silverstein KA, Lajoie M, Bertrand D, El-Mabrouk N, et al. The artiodactyl APOBEC3 innate immune repertoire shows evidence for a multi-functional domain organization that existed in the ancestor of placental mammals. BMC Mol Biol 2008;9:104.

36. Refsland EW, Stenglein MD, Shindo K, Albin JS, Brown WL, Harris RS. Quantitative profiling of the full APOBEC3 mRNA repertoire in lymphocytes and tissues: Implications for HIV-1 restriction. Nucleic Acids Res 2010;38:4274-84.

37. Koning FA, Newman EN, Kim EY, Kunstman KJ, Wolinsky SM, Malim MH. Defining APOBEC3 expression patterns in human tissues and hematopoietic cell subsets. J Virol 2009;83:9474-85.

38. Yamanaka S, Balestra ME, Ferrell LD, Fan J, Arnold KS, Taylor S, et al. Apolipoprotein B mRNA-editing protein induces hepatocellular carcinoma and dysplasia in transgenic animals. Proc Natl Acad Sci USA 1995;92:8483-7.

39. Saraconi G, Severi F, Sala C, Mattiuz G, Conticello SG. The RNA editing enzyme APOBEC1 induces somatic mutations and a compatible mutational signature is present in esophageal adenocarcinomas. Genome Biol 2014;15:417.

40. Petersen-Mahrt SK, Harris RS, Neuberger MS. AID mutates
E. coli suggesting a DNA deamination mechanism for antibody diversification. Nature 2002;418:99-103.

41. Ding Q, Chang CJ, Xie X, Xia W, Yang JY, Wang SC, et al. APOBEC $3 \mathrm{G}$ promotes liver metastasis in an orthotopic mouse model of colorectal cancer and predicts human hepatic metastasis. J Clin Invest 2011;121:4526-36.

42. Okazaki I, Hiai H, Kakazu N, Yamada S, Muramatsu M, Kinoshita K, et al. Constitutive expression of AID leads to tumorigenesis. J Exp Med 2003;197:1173-81

43. Robbiani DF, Nussenzweig MC. Chromosome translocation, B cell lymphoma, and activation-induced cytidine deaminase. Annu Rev Pathol 2013;8:79-103.

44. Ramiro AR, Jankovic M, Eisenreich T, Difilippantonio S, Chen-Kiang S, Muramatsu M, et al. AID is required for c-myc/IgH chromosome translocations in vivo. Cell 2004;118:431-8.

45. Lackey L, Demorest ZL, Land AM, Hultquist JF, Brown WL, Harris RS. APOBEC3B and AID have similar nuclear import mechanisms. J Mol Biol 2012;419:301-14.

46. Land AM, Law EK, Carpenter MA, Lackey L, Brown WL, Harris RS. Endogenous APOBEC3A DNA cytosine deaminase is cytoplasmic and nongenotoxic. J Biol Chem 2013;288:17253-60.

47. Roberts SA, Lawrence MS, Klimczak LJ, Grimm SA, Fargo D, Stojanov $\mathrm{P}$, et al. An APOBEC cytidine deaminase mutagenesis pattern is widespread in human cancers. Nat Genet 2013;45:970-6.

48. Cancer Genome Atlas Research Network. Comprehensive molecular characterization of urothelial bladder carcinoma. Nature 2014;507:315-22.

49. Rebhandl S, Huemer M, Gassner FJ, Zaborsky N, Hebenstreit D, Catakovic $\mathrm{K}$, et al. APOBEC3 signature mutations in chronic lymphocytic leukemia. Leukemia 2014;28:1929-32.

50. Henderson S, Chakravarthy A, Su X, Boshoff C, Fenton TR APOBEC-mediated cytosine deamination links PIK3CA helical domain mutations to human papillomavirus-driven tumor development. Cell Rep 2014;7:1833-41.

51. Krokan HE, Sætrom P, Aas PA, Pettersen HS, Kavli B, Slupphaug G. Error-free versus mutagenic processing of genomic uracil-relevance to cancer. DNA Repair 2014;19:38-47.

52. Chan K, Resnick MA, Gordenin DA. The choice of nucleotide inserted opposite abasic sites formed within chromosomal DNA reveals the polymerase activities participating in translesion DNA synthesis. DNA Repair 2013;12:878-89.

53. Kidd JM, Newman TL, Tuzun E, Kaul R, Eichler EE. Population stratification of a common APOBEC gene deletion polymorphism. PLoS Genet 2007;3:e63.

54. Komatsu A, Nagasaki K, Fujimori M, Amano J, Miki Y. Identification of novel deletion polymorphisms in breast cancer. Int $\mathrm{J}$ Oncol 2008;33:261-70

55. Xuan D, Li G, Cai Q, Deming-Halverson S, Shrubsole MJ, Shu XO, et al. APOBEC3 deletion polymorphism is associated with breast cancer risk among women of European ancestry. Carcinogenesis $2013 ; 34: 2240-3$

56. Long J, Delahanty RJ, Li G, Gao YT, Lu W, Cai Q, et al. A common deletion in the APOBEC3 genes and breast cancer risk. J Natl Cancer Inst 2013;105:573-9.

57. 1000 Genomes Project Consortium, Abecasis GR, Altshuler D, Auton A, Brooks LD, Durbin RM, Gibbs RA, et al. A map of 
human genome variation from population-scale sequencing. Nature 2010;467:1061-73.

58. Nik-Zainal S, Wedge DC, Alexandrov LB, Petljak M, Butler AP, Bolli N, et al. Association of a germline copy number polymorphism of APOBEC 3A and APOBEC3B with burden of putative APOBEC-dependent mutations in breast cancer. Nat Genet 2014;46:487-91

59. Koning FA, Goujon C, Bauby H, Malim MH. Target cell-mediated editing of HIV-1 cDNA by APOBEC 3 proteins in human macrophages. J Virol 2011;85:13448-52.

60. Chen H, Lilley CE, Yu Q, Lee DV, Chou J, Narvaiza I, et al APOBEC 3A is a potent inhibitor of adeno-associated virus and retrotransposons. Curr Biol 2006;16:480-5.

61. Zur Hausen H. Papillomaviruses in the causation of human cancers-a brief historical account. Virology 2009;384:260-5.

62. Harris RS. Enhancing immunity to HIV through APOBEC. Nat Biotechnol 2008;26:1089-90. 